\title{
Disease-associated malnutrition in the year 2000
}

\author{
C R Pennington
}

\begin{abstract}
Summary
Malnutrition is associated with increased morbidity and mortality and is common in patients admitted to hospital. Nutritional status is not routinely assessed on admission, and nutritional depletion escapes recognition in the majority of affected patients. Nutritional status declines during hospital stay, and this trend is most marked in patients who are already malnourished on admission. Techniques for nutritional support are available, their appropriate use leads to improved nutritional status and clinical outcome in most patients. There is evidence that the current use of artificial nutrition is suboptimal and associated with a high complication rate. The introduction of clinical guidelines and the formation of nutrition support teams will improve nutritional management in the future. The development of new substrates and 'pharmaconutrition' is likely to further improve the outcome for many patients. There will remain a need for more studies to define the cost efficacy of artificial nutrition across a broad spectrum of clinical practice.
\end{abstract}

Keywords: malnutrition; artificial nutrition

Department of Gastroenterology,

Ninewells Hospital and Medical School,

Dundee, Scotland, UK

C R Pennington

Accepted 10 June 1997
During the last 20 years, studies of the nutritional status of hospital patients have consistently found that undernutrition is common. Early reports from the USA and UK described a prevalence of $50 \%$ in patients in medical and surgical wards. ${ }^{1-3}$ During the last 16 years, several studies have investigated the incidence of malnutrition in patients on admission to hospital. ${ }^{4-10}$ With the exception of a small study in which selected patients were examined, ${ }^{6}$ the incidence of undernutrition was found to range from $20-40 \%$ (tables 1 and 2). A recent study of 500 admissions, ${ }^{9} 100$ consecutive admissions to each of the main specialties of general medicine, general surgery, care of the elderly, respiratory medicine and orthopaedic surgery, found an overall incidence of malnutrition of $40 \%$, with moderate or severe malnutrition in $27 \%$. Furthermore there is evidence that disease-associated malnutrition escapes recognition. In an early study, nutritional information was recorded in the case notes of only $21 \%$ of all patients. ${ }^{3}$ More recently, it was noted that only $48 \%$ of the nutritionally depleted patients had any nutritional information in their medical records during their hospital course. ${ }^{9}$ This probably explains why the majority of affected patients were not treated. In this study weight loss occurred in $65 \%$ of the 112 patients who were in hospital for more than one week and were re-assessed on discharge; 55 had malnutrition identified on admission and only 10 were referred for nutritional support of whom seven gained weight. In contrast, $75 \%$ of the other 45 malnourished patients continued to deteriorate nutritionally.

Thus, disease-related malnutrition is common, escapes recognition, and becomes worse during the hospital stay except in the minority of patients who are referred for nutritional management. This implies that disease-associated malnutrition is not considered significant. A study in which 450 nurses and 319 junior doctors were surveyed in 70 hospitals revealed that only $34 \%$ of the doctors knew if their patients had been weighed, while $60 \%$ of the remainder considered weight to be unimportant. ${ }^{11}$ Such findings suggest that the subject of clinical nutrition is not adequately addressed in the undergraduate curriculum or during clinical training.

These studies raise several questions: what is meant by malnutrition, how is it measured, why does it occur, what is its significance to the patient and the community, and how should it be treated?

\section{Measurement of malnutrition}

Unfortunately we lack adequate tools for the measurement of this condition. Body mass index (BMI), the weight in $\mathrm{kg}$ divided by the height in metres ${ }^{2}$ is the gold standard for defining nutritional category. A BMI of 19 or below signifies undernutrition, and 25 or above excessive weight. Measurement of mid-arm muscle circumference and triceps skin fold thickness will help to identify tissue wasting of muscle and fat. Serial measurements of weight and anthropometry can be used to chart nutritional progress under some circumstances over prolonged periods. ${ }^{12}$ However, reliance on weight and structural change measured in this way is frequently inadequate and may be misleading. Impairment of organ function develops with nutritional depletion before significant structural change can be measured, and nutritional deprivation is especially likely to be overlooked in the obese patient during hospital illness. Thus such measurements need to be interpreted in relation to the diet over the previous few weeks. Furthermore parameters such as weight and arm circumference are influenced by hydration status which varies considerably during the course of significant illness, and the techniques of anthropometry are prone to inter-observer error. Bioelectrical impedance analysis can provide more accurate information about body composition in the normal subject, however, disturbance of water and electrolyte metabolism in ill patients and patients with organ failure limit the usefulness of this technique. ${ }^{12}$ 


\begin{tabular}{|l|}
\hline Nutritional screening \\
\hline Questions \\
- reduction in food consumption \\
- normal weight \\
Measurententional weight loss \\
- weight \\
- height \\
\hline
\end{tabular}

Box 1

\begin{tabular}{|l|}
\hline Some causes of \\
disease-associated \\
malnutrition \\
\hline Anorexia \\
- depression \\
- chronic disease \\
Inability to eat \\
- neurological disorders \\
- oro-pharyngeal disease \\
Intestinal disease \\
- inflammatory bowel disease \\
- radiation enteritis \\
gluten enteropathy \\
- hollow visceral myopathy \\
Inflammatory response to infection and \\
trauma \\
- cytokines \\
\hline
\end{tabular}

Box 2

\begin{tabular}{|l|}
\hline Some examples of the \\
metabolic effects of cytokines \\
\hline - anorexia \\
- pyrexia \\
release of amino acids from muscle \\
- insue \\
increased glucose transport \\
- promulation of hepatic lipid synthesis \\
synthesis of acute phase protein \\
- reduced albumin synthesis \\
- increased vascular permeability \\
\hline
\end{tabular}

Box 3
Table 1 Prevalence of malnutrition in hospital patients

\begin{tabular}{lllll}
\hline Ref & Year & Patients $(n)$ & Type of patients & \% malnourished \\
\hline 1 & 1974 & 131 & General surgical & 50 \\
2 & 1976 & 251 & General medical & 44 \\
3 & 1977 & 105 & General surgical & 50 \\
\hline
\end{tabular}

Older studies employed albumin and other serum protein measurements. The concentration of these synthetic proteins is influenced by the acute phase response, and changes in hydration and vascular permeability. While hypoalbuminaemia is a marker of surgical risk, it is not a true indicator of nutritional status, and normal serum concentrations are maintained in starving patients who are not stressed, for example, in anorexia nervosa, until the terminal phases of illness. ${ }^{13}$

There is a need for functional measurements of nutritional status. Hand grip dynamometry and pulmonary function tests are employed by some as measurements of muscle function, both have obvious limitations, including the need for patient co-operation and the presence of underlying pulmonary disease.

Thus, many authorities advocate simple nutritional screening which will identify patients who are either at risk, or who have established nutritional depletion and who merit nutritional assessment (box 1). Nutritional screening can be undertaken as part of the admission process, perhaps by the nursing staff. ${ }^{14}$ One scheme suggests that the measurement of weight and height, and information about unintentional weight loss, reduction in food consumption, and normal weight should be obtained in every patient. ${ }^{11}$ Patients who are identified as being at risk can then be assessed with a more detailed dietary history complemented by anthropometric measurements.

\section{Causes of disease-associated malnutrition}

The development of disease may be accompanied by many factors which cause malnutrition (box 2). Depression and chronic illness may lead to anorexia. Patients with neurological disorders such as cerebrovascular disease and motor neuron disease may be unable to eat. Intestinal disease such as gluten enteropathy, Crohn's disease, radiation enteritis, and motility disorders, reduce the efficacy of the intestine and are frequently accompanied by maldigestion and the impaired absorption of nutrients.

The additional influences of infections, burns, and trauma, including surgical operations, may lead to accelerated nutritional decline. Although this has been explained by a presumed increase in energy requirements in the stressed patient, and the humoral response to stress, most of the observed changes are attributable to the release of cytokines. Energy requirements in critical illness have previously been overestimated, any increased energy needs are matched by a reduction in mobility. ${ }^{15}$ Cytokines such as interleukin, tumour necrosis factor, and interferons are principally responsible for the metabolic changes. They share multiple activities (box 3) and cause anorexia, pyrexia, release of amino acids from muscle tissue, increased glucose transport, and reduced albumin synthesis, and increased vascular permeability. ${ }^{16}$ Furthermore, in severely ill patients, especially those in intensive care, the effect of the initial pathology may be compounded by the secondary effect of the breakdown in the gut mucosal barrier, with increased permeability to microbial toxins, and enhanced translocation of bacteria, continuing cytokine generation and leading to greater tissue and organ damage. ${ }^{17}$

The feeding of patients in hospital has been reviewed. ${ }^{18}$ Attention has been drawn to many important factors which collectively deprive the patient of

Table 2 Incidence of malnutrition on admission to hospital

\begin{tabular}{lllll}
\hline Ref & Year & $\begin{array}{l}\text { No of patients } \\
(n)\end{array}$ & Type of patients & \% malnourished \\
\hline 4 & 1980 & 200 & General medical, general surgical & 32 \\
5 & 1983 & 744 & Orthopaedic surgical & 53 \\
6 & 1989 & 84 & General surgical & 14 \\
7 & 1990 & 501 & Care of elderly & 19 \\
8 & 1993 & 200 & General medical & 20 \\
9 & 1994 & 500 & $\begin{array}{l}\text { General medical, general surgical, } \\
\text { orthopaedic surgical, respiratory medicine, } \\
\text { care of elderly }\end{array}$ & 40 \\
10 & 1996 & 129 & Intensive care & 43 \\
\hline
\end{tabular}




\begin{tabular}{|l|}
\hline Some of the effects of \\
disease-associated \\
malnutrition \\
\hline Impaired mental function \\
- apathy \\
- fatigue \\
- inability to co-operate with treatment \\
Impaired muscle function \\
- respiratory failure \\
Impaired immune function \\
- increased incidence of infection \\
\hline
\end{tabular}

Box 4

\begin{tabular}{|l|}
\hline $\begin{array}{l}\text { Methods of artificial } \\
\text { nutritional support }\end{array}$ \\
\hline Enteral nutrition \\
$\bullet$ oral supplements \\
enteral tube feeding: nasogastric, \\
nasojejunal; gastrostomy (most \\
commonly PEG tubes), jejunostomy \\
(via PEG, or surgical placement) \\
Parenteral nutrition \\
- peripheral \\
central \\
\hline
\end{tabular}

Box 5 adequate nutrition. These include inappropriate dietary choice by the patient, the interruption of meals by staff, missed meals through therapeutic or investigative procedures, and the lack of flexibility in hospital catering.

Thus sufficient food may be unavailable, some patients experience difficulty in eating, intestinal function is often impaired, and these effects can be compounded by the influence of inflammatory mediators.

\section{The effect of disease-associated malnutrition}

Malnutrition impairs organ function (box 4). Muscles become weaker and fatigue more rapidly. These effects can be demonstrated in normal people during dietary restriction of only two weeks duration. ${ }^{19}$ Not only may such changes effect the mobilisation of patients after operative procedures, they may also contribute to respiratory and cardiac failure. Protein energy malnutrition in children is associated with thymic atrophy and similar changes affect other lymphoid tissues. There is a failure of antibody production and phagocyte function. ${ }^{20}$ Thermoregulation is impaired, and mental function can be affected, patients become withdrawn and apathetic. Some patients demonstrate features of specific nutrient deficiencies. These include thiamine and Wernicke's encephalopathy, folate and megaloblastic anaemia, and ascorbic acid and scurvy. Growth failure occurs in children. These effects contribute to poor outcome from disease and following surgery. Prolonged hospital stay and increased morbidity and mortality have been documented in association with malnutrition.

In a study of 80 patients who were undergoing surgery, protein depletion, determined by neutron activation analysis, was documented in 39 patients. The depleted patients, who were similar in other respects to the non-depleted group, had impaired respiratory function, increased propensity to pneumonia, and prolonged hospital stay. ${ }^{21}$ Malnutrition was identified in 55 of 129 patients who were admitted to an intensive care unit. The incidence of complications and the number of patients who were not discharged from hospital were both greater in patients who were malnourished on admission, and the impact of malnutrition was greater in the patients who were less ill. ${ }^{10}$ The increased operative mortality in malnourished patients was described 60 years ago. ${ }^{22}$

\section{Prevention and treatment of disease-associated malnutrition}

Artificial nutritional support is used to prevent or treat malnutrition. Artificial nutrition may be given as enteral feeding, either oral supplements or enteral tube feeding, and parenteral nutrition which can be administered by peripheral or central veins (box 5).

\section{ENTERAL FEEDING}

Enteral feeding has several advantages over parenteral feeding. Firstly, it stimulates the gut-associated immune function. ${ }^{17}$ The intestinal mucosa is partly dependent on luminal nutrition, and glutamine is an important fuel for the enterocyte; this amino acid is not provided in most routinely available parenteral nutrition solutions because of limited stability. Glutamine may also be important in relation to the intestinal barrier function, for the prevention of microbial translocation. ${ }^{17}$ Enteral feeding can stimulate intestinal and biliary motility. Consequently, feeding by the oral or enteral route has physiological advantages. In addition, it is much cheaper and safer than parenteral nutrition. A study in which trauma patients were randomised to enteral or parenteral nutrition reported pneumonia and intra-abdominal abscesses, respectively, in $31 \%$ and $13.4 \%$ of those patients who received parenteral feeding, the corresponding figures for patients who were given enteral feeding were $11.8 \%$ and $1.9 \% .{ }^{23} \mathrm{~A}$ meta-analysis of all randomised controlled trials of enteral and parenteral nutrition revealed more infection in the patients who were fed parenterally. ${ }^{24}$ This may in part reflect deterioration in the gut barrier function during parenteral nutrition. Wherever possible, some nutritional support should be given by the enteral route. The recognition that intestinal function returns early in the postoperative period has lead to the use of jejunostomy feeding in many patients who would previously have required parenteral nutrition.

For patients without gastric stasis, nasogastric feeding in the short term, or percutaneous gastrostomy (PG) in the longer term, facilitate nutritional management. PG has been a useful development for patients with chronic swallowing disorders, such as motor neuron disease and cerebrovascular disease. Although associated with a procedure-related risk, PG ensures improved nutritional management and is preferred by patients who have previous experience of nasogastric tubes. ${ }^{25}{ }^{26}$ Most PG tubes are inserted by the endoscopic technique, 


\section{Examples of important complications of parenteral nutrition}

Metabolic and nutritional complications

- disorders of glucose and electrolyte balance

- nutrient excess: glucose and hepatic steatosis

- nutrient deficiency: selenium and cardiomyopathy

Catheter-related complications

- catheter infection: local or systemic

- catheter occlusion: lipid or fibrin

- vein thrombosis

- air embolism

Effect of treatment on other organ systems

- hepatobiliary disease

- immune suppression

- bone disease

Box 6 however direct placement under radiological imaging offers an alternative for those patients with an obstructing oesophageal lesion.

\section{PARENTERAL NUTRITION}

Parenteral nutrition is reserved for those patients in whom intestinal function is not available or inadequate. It is expensive and can be associated with significant complications (box 6). ${ }^{27}$ The most serious catheter complications include catheter-related sepsis and central vein thrombosis. The effect on the hepatobiliary system may in part reflect the lack of oral feeding with the failure to stimulate gall bladder contraction leading to the formation of biliary sludge and gallstones. The adverse effect on the intestinal mucosal integrity with microbial translocation and absorption of toxins may be another indirect effect due to the lack of conditionally essential nutrients such as glutamine. Inappropriate nutrient provision, excess of glucose and some amino acids, and inadequate provision of some trace elements such as selenium may also be deleterious. However, with the exception of infection, most of the complications occur only with prolonged treatment.

Many of these disadvantages can be prevented with expert management. This is best provided with a multidisciplinary nutrition team, ${ }^{28}{ }^{29}$ currently these teams are only available in a minority of hospitals. ${ }^{30}$

In the majority of cases, parenteral nutrition prescribed in hospital is for less than two weeks. Under these circumstances access should be by the peripheral vein which avoids many of the problems associated with central vein catheters. Ultra fine bore $15-\mathrm{cm}$ catheters are an alternative to conventional canulae. With proper care, canulae may last 10 days. However, regular inspection of the peripheral veins is essential, and the canula must be removed at the first sign of inflammation. Some authorities recommend nitrate patches over the vein, and low-dose heparin and hydrocortisone in the nutrition solution. ${ }^{31} \mathrm{~A}$ few centres employ peripherally inserted central catheters. While such devices avoid complications associated with central catheter placement, the safety of and place for these catheters remain to be established.

ARTIFICIAL NUTRITION IN THE STARVING PATIENT AND IN THE CRITICALLY ILI

Nutritional support in the starving patient will lead to an anabolic response. In the severely depleted patient the switch from endogenous lipid to exogenous carbohydrate may be one explanation for the dramatic reduction in the serum concentrations of phosphate, potassium, and magnesium which move into the cell under the influence of insulin. Under these circumstances, additional supplements will be required to prevent potentially fatal complications such as hypophosphataemia. ${ }^{32}$

Nutritional support in the stressed patient will not reverse the nutritional depletion. Although an increase in protein synthesis can be achieved, it will not reduce the rate of protein breakdown. Thus, the process of nutritional depletion will be slowed but not reversed. ${ }^{33}$ Methods to improve the metabolic response in such patients have included the use of novel substrates and growth hormone. These will be discussed later.

Nutritional depletion takes a long time to reverse, and the process may only be retarded in the very ill patient. Consequently it is recommended that no patient should be completely deprived of nutrition for more than seven days, and that support should commence earlier in the patient who is either stressed, or already malnourished.

\section{THE EFFECT OF ARTIFICIAL NUTRITION ON NUTRITIONAL STATUS}

We recently conducted a study in which malnourished patients were allowed free access to hospital food and randomised on admission to hospital to one of three groups. The control group received no specific nutritional management. One intervention group received oral supplements, and the other group were given supplementary nocturnal nasogastric feeding. Supplements were prescribed to complement the oral diet so as to meet the estimated energy requirements. The consumption of food was similar in all three groups, but weight gain occurred in the two intervention groups, while the control group continued to lose weight. ${ }^{34}$

It has been shown that nutritional status can be improved in elderly patients by increasing the energy density of hospital food. ${ }^{35}$ This may be the most costeffective approach for patients who are able to eat. Nutritional recovery may be slow and incomplete, especially in the elderly with an acute phase response. The intestinal tract may not always be available, especially in the surgical patient. In a study of patients who had lost weight on account of malignant disease, postoperative parenteral nutrition prevented a further decline in muscle protein in association with surgery. ${ }^{36}$ 
THE EFFECT OF ARTIFICIAL NUTRITION ON CLINICAL OUTCOME

Clearly the most important end-point is recovery from disease. There have been many studies which established the role of artificial nutrition in different patient populations. The early use of oral supplements reduced the incidence of complications following major gastrointestinal surgery. ${ }^{37}$ Supplemental nasogastric feeding lead to reduced mortality and a reduction in hospital stay in malnourished orthopaedic patients with a fractured neck of femur. ${ }^{38}$ Oral supplements also reduced both the complication rate, including bed sores and cardiac failure, and the mortality in another group of elderly patients with fracture of the neck of femur, an effect that was sustained for six months after the injury. ${ }^{39}$ A large study of elderly patients demonstrated that the use of an oral supplement greatly reduced mortality at six months. ${ }^{7}$

The value of peri-operative nutritional support was assessed by giving depleted patients 10 days of enteral or parenteral nutrition. There were depleted and non-depleted control groups. The depleted controls had more complications than the non-depleted controls. Artificial nutrition caused a significant reduction in the major complications in patients who had lost more than $10 \%$ of their body weight. ${ }^{40}$ The use of immediate postoperative parenteral nutrition, as opposed to commencing parenteral nutrition at seven days if patients were unable to take oral diet at that time, was assessed in 35 patients who had undergone radical cystectomy. Early parenteral nutrition was associated with an average reduction of hospital stay of seven days. ${ }^{41}$

The fact that there is no benefit from the routine use of artificial nutrition in patients who are not malnourished and not at risk of nutritional depletion has also been well established. ${ }^{42}$ Such practice is ineffective, wasteful, and harmful.

MANAGEMENT OF ARTIFICIAL NUTRITION

There is evidence that the delivery of artificial nutrition is suboptimal. Many studies attest to the high complication rate which is potentially avoidable, especially with effective nutrition support teams. In spite of recent trends, the majority of the UK hospitals lack such a facility. ${ }^{30}$ A six-month study of artificial nutrition in one of these hospitals revealed that less than half of the patients received their estimated needs with either parenteral or enteral nutrition. The problem arose because errors of prescription, the use of standard regimes, and problems of delivery, including a $10 \%$ catheter infection rate. ${ }^{43}$

\section{Disease-associated malnutrition: prospects for the year 2000}

There is an increasing recognition that standards of care vary between different centres and different countries. This has led to a trend to introduce uniform and optimum standards in the management of a range of disorders, including the treatment of cancer. This activity has been stimulated by the wide application of clinical audit, and the acceptance of the need for evidence-based medicine.

Many specialist groups are developing guidelines and standards. These groups include the American Society for Parenteral and Enteral Nutrition, the British Association for Parenteral and Enteral Nutrition, and the British Society of Gastroenterology. The guidelines are likely to influence clinical practice. They cover the recognition of nutritional depletion and standards of management.

\section{THE INCREASED USE OF ARTIFICIAL NUTRITION}

Current data suggest that $10 \%$ of hospital patients in the UK receive artificial nutrition. There is evidence that $30 \%$ of patients suffer from clinically significant disease-associated malnutrition; this may be an underestimate, and many patients who are normally nourished on admission could benefit from artificial nutrition to prevent nutritional depletion during therapeutic intervention. Consequently, there is considerable scope for increasing the use of this form of treatment. However, financial limitations mean that healthcare rationing is a reality in all healthcare systems, even if it is not acknowledged. Funding for improved nutritional care will need to be sought against competing interests. Undoubtedly, improved organisation of the way in which artificial nutrition is administered will release some funding. This may be achieved by reducing the incidence of expensive complications, and ensuring appropriate selection of the type of treatment, for example, by prescribing enteral feeding where possible and minimising the use of expensive parenteral nutrition. Furthermore, overall healthcare costs, including the length of hospital stay and the period of convalescence, may be expected to reduce with optimum nutritional management. Nevertheless, there is an urgent need for more information about the cost efficacy of this form of treatment to determine the appropriate allocation of resources. 
THE ORGANISATION OF ARTIFICIAL NUTRITION

Early data from the US demonstrated a significant reduction in the high complication rate during artificial nutrition following the introduction of nutrition support teams. ${ }^{28}$ Similar results can be achieved through the adoption of protocols introduced by the teams, and the growth in such teams in the US has stopped. However, the problem is not simply one of minimising complication rates during nutritional management. The broader issues include the need to ensure that nutritional depletion and risk factors are recognised, that appropriate treatment is given, and that outcomes are monitored. Data suggest that guidelines from local nutrition advisory groups are not implemented, ${ }^{43}$ and thus some form of intervention is needed. This may apply until nutritional education and training programmes become established. During the last few years, the number of British hospitals with nutrition support teams has increased, teams now being available in $60 \%$ of teaching centres. ${ }^{30}$ This trend is likely to continue, influenced by increased public awareness and staff education. The nature of the team and the method of operation will depend on local circumstances. The role may be restricted to the implementation of policy determined by the nutrition advisory group, with the responsibility for treatment remaining with the supervising consultant. Each team is likely to contain a clinician, nurse, dietician, and pharmacist. In larger hospitals there may be different teams for specific areas, eg, paediatrics and intensive care, with a supervising nutritional advisory group comprising key representatives of each relevant discipline and management.

TEACHING AND TRAINING

The subject of nutrition is not adequately addressed in the medical curriculum of many medical schools in the UK. ${ }^{44}$ Furthermore, until recently it has not been included in postgraduate training programmes. These issues have recently been addressed, and the inclusion of nutrition as a core subject in medical curricula seems inevitable. Clearly this involves much more than simply nutritional support. The role of nutrition in the development and treatment of disease can be addressed in relation to each specialty, or in part of the curriculum devoted to nutrition. The latter may be more satisfactory, but the former is more readily achieved. Societies such as the British Society of Gastroenterology have recently included artificial nutrition in their recommendations for core training of the specialist registrar. Thus, by the year 2000 , senior doctors will be familiar with artificial nutrition and related subjects on appointment.

RESEARCH AND DEVELOPMENT

Further information will be available in at least two areas: the clinical role of more recently developed substrates, ${ }^{12}$ and the value and cost of artificial nutrition in a range of clinical settings.

Glutamine has recently been introduced in the form of a dipeptide for addition to standard parenteral nutrition solutions. Glutamine is a conditionally essential amino acid which is omitted from standard amino acid solutions because of concerns about stability. It appears to have a beneficial role in sustaining the function of fast turnover tissues such as the intestinal epithelium and white cells. It increases intracellular volume, glycogen storage, and growth hormone release. The evidence that it has a clinical benefit, in part due to the protection of the integrity of the intestinal mucosa, is growing. By the year 2000 this substrate is likely to be widely used in the management of the critically ill patient. The role of other substrate manipulations, eg, high-dose arginine, to stimulate the immune response is less clear. Similarly, the need for mixtures of medium- and long-chain fatty acids or structured lipids in parenteral nutrition solutions, to reduce the production of pro-inflammatory mediators in the stressed patient, and the inclusion of nucleotides in enteral products, are likely to remain under investigation.

The early administration of an enteral formula supplemented with arginine, nucleosides, and fish oil in a multicentre study of 326 intensive care patients did not affect mortality. However, in those patients who were able to tolerate at least $821 \mathrm{ml}$ per day, there was an average eight-day reduction in the length of hospital stay. ${ }^{45}$ More work is required before such products are used in routine practice. The same is probably true of the 'disease-specific' enteral nutritional products for which the evidence for clinical benefit is unlikely to justify the increased cost. More data will also be available on the potential roles of growth hormone and insulin-like growth factor 1 in nutritional management in both sick and starving patients. Increasing and improved data on the role and cost of artificial nutrition will help to define the use of this treatment in various clinical circumstances which will, in turn, facilitate funding decisions.

These topics call for large multicentre studies. Such studies are easier when patients with a specific problem are managed by an identified consultant and 
when the pharmaceutical treatment for the condition is commercially lucrative, facilitating sponsorship. As neither of these conditions apply to artificial nutrition, progress is likely to be slow and meaningful studies will be difficult to fund.

\section{A SPECIALTY OF CLINICAL NUTRITION}

Nutrition plays a crucial role in the pathogenesis and management of disease. Inadequate, excessive, and inappropriate nutrition all lead to increased morbidity and mortality. Nutritional factors have been linked with a range of disorders, including hypertension, vascular disease, and cancer. Dietary management is important in the treatment of many disorders, including ischaemic heart disease, diabetes, and renal disease. Consequently, there is a strong case for the development of the specialty of nutrition, as currently exists in some European countries. Within the UK, this will be difficult to achieve, not least because of the rigidity of the specialty-based training programmes and requirements, and the lack of opportunities for trainees with unconventional training experience. Nevertheless, the development of academic departments of nutrition, and moves within specialist societies, such as the Nutritional Society, suggest that the goal is attainable in the long term-but not by the year 2000 !

1 Bistrian BR, Blackburn GL, Hallowell E, Heddle R. Protein status of general surgical patients. $\mathscr{F} A M A \quad 1974 ; 235: 858-60$.

2 Bistrian BR, Blackburn GL, Vitale J, Cochran $\mathrm{D}$, Naylor J. Prevalence of malnutrition in general medical patients. $\mathscr{f} A M A$ 1976;235: 1567-70.

3 Hill GL, Blackett RL, Pickford I, et al. Malnutrition in surgical patients - an unrecognised problem. Lancet 1977;i:689-92.

4 Willard MD, Gilsdorf RB, Price RA. Proteincalorie malnutrition in a community hospital. calorie malnutrition in a
$\exists A M A$
$1980 ; 243: 1720-2$.

5 Bastow MD, Rawlings J, Allison SB. Undernutrition, hypothermia and injury in elderly women with fracture neck of femur: an injury response to altered metabolism? Lancet 1983;i: 143-5.

6 Zador DA, Truswell AS. Nutritional status on admission to a general surgical ward in a Sydney hospital. Aust NZ F Med 1987;17:234-40.

7 Larsson J, Unosson N, Ek AC, Nilsson L, Thorslund S, Bjurulf P. Effect of dietary supplement on nutritional status and clinical outcome in 501 geriatric patients - a randomised study. Clin Nutr 1990;9:179-84.

8 Cederholm T, Jagren C, Hellstrom K. NutriCederholm T, Jagren C, Hellstrom K. Nutritional status and performance capacity in inter-

9 McWhirter JP, Pennington CR. The incidence and recognition of malnutrition in hospital. $B M \mathcal{F}$ 1994;308:945-8.

10 Giner M, Laviano A, Meguid MM, Gleason JR. In 1995 a correlation between malnutrition and poor outcome in critically ill patients still exists. Nutrition 1996;12:23-9.

11 Lennard-Jones JE, Arrowsmith H, Davison C, Denham AF, Micklewright A. Screening by nurses and junior doctors to detect malnutrition when patients are first assessed in hospital. Clin when patients are first

12 Pennington CR. Disease and malnutrition in Pritish Hospitals. Proc Nutr Soc 1997;56:1-15.

13 Anderson CF, Wochers DN. The utility of Anderson CF, Wochers DN. The utility of
serum albumin values in the nutritional assessment of hospitalised patients. Mayo Clin Proc 1982;57:181

14 Reilly EN, Martineau JK, Moran A, Kennedy H. Nutritional screening - evaluation and implementation of a simple nutritional risk score. Clin Nutr 1995;14:269-74.

15 Elia $M$. Changing concepts of nutrient requirements in disease: implications for artificial nutritional support. Lancet 1995;345:1279-84.

16 Tracy KJ. TNF and other cytokines in the metabolism of septic shock and cachexia Clin Nutr 1992;11:1-11.
17 Gardiner KR, Kirk SJ, Rowlands BJ. Novel substrates to maintain gut integrity. Nutr Res Rev 1995;8:43-66.

18 McGlone PC, Dickison JWT, Davis GJ. The feeding of patients in hospital: a review. $\mathcal{F} R$ Soc Health 1995;282-8.

19 Jeejeebhoy KN. Bulk or bounce - the object of nutritional support. $\mathcal{F}$ Parenteral Enteral Nutr 1988;12:539-45.

20 Animashaun A, Heatley RV. Nutritional immunology. In: Heatley RV, Green JH, Losowsky nology. In: Heatley RV, Green JH, Losowsky MS, eds, Consensus in clinical nutrition. pambridge:

21 Windsor JA, Hill GL. Risk factors for post operative pneumonia. The importance of protein depletion. Ann Surg 1988;17:181-5

22 Studley HO. Percentage of weight loss - a basic indicator of surgical risk in patients with chronic peptic ulcer. $\mathscr{F} A M A$ 1936;106:458-60.

23 Kudsk KA, Croce MA, Fabian TC, et al. Enteral vesus parenteral feeding. Ann Surg 1992;215: 503-13.

24 Moore FA, Feliciano DV, Andrassy RJ, et al. Early enteral feeding, compared with parenteral, reduces post-operative septic complications. reduces post-operative septic complications. The results of

25 Wicks C, Gimson A, Lavianos V, et al. Assessment of percutaneous endoscopic gastrostomy feeding tube as part of an integrated approach to enteral feeding. Gut 1992;33:613 6.

26 Park RHR, Allison MC, Lang J, Russell R. Randomised comparison of percutaneous endoscopic gastrostomy and nasogastric tube feeding in patients with persisting neurological dysphagia. $B M \mathcal{F}$ 1992;304:1406-9.

27 Pennington CR. Parenteral nutrition: the management of complications. Clin Nutr 1991;10. 133-7.

28 Burnham WR. The role of the nutrition support team. In: Payne-James J, Grimble G, Silk D, eds, Artificial nutritional support in clinical practice. London: Edward Arnold, 1995; pp 175-186.

29 Gales BJ, Gales MJ. Nutrition support teams: review of comparative trials. Ann Pharmacothe 1994;28:227-35.

30 Payne-James J, De Gara CJ, Grimble GK, Silk DBA. Articial nutritional support in hospitals in the United Kingdom - 1994: 3rd National Survey. Clin Nutr 1995;14:329-35.

31 Tighe MJ, Wong C, Martin IG, McMahon MJ Do heparin, hydrocortisone, and glyceryl trinitrate influence thrombophlebitis during full trate influence thrombophlebitis during full Parenteral Enteral Nutr 1995;19:507-9.
32 Solomon SN, Kirby DS. The refeeding syndrome: a review. $\mathcal{F}$ Parenteral Enteral Nutr 1990;14:90-5.

33 Askanazi J, Carpentier YA, Elwyn DH, et al. Infuence of total parenteral nutrition on fuel utilisation in injury and sepsis. Ann Surg 1980;191:40-6.

34 McWhirter JP, Pennington CR. A comparison between oral and nasogastric supplements in malnourished patients. Nutrition 1996;12:5026.

35 Olin AO, Osterberg B, Hadell K, Armyr I, Jerstrom S, Ljungqvist O. Energy enriched hospital food to improve energy intake in elderly patients. F Parenteral Enteral Nutr 1996;20:93-7.

36 Petersson B, Hultzman E, Andersson K, Wernerman J. Human skeletal muscle protein: effect of malnutrition, elective surgery and total parenteral nutrition. Clin Sci 1995;88:479-84.

37 Keele AM, Bray M, Emery P, Duncan H, Silk D. Two phased randomised controlled clinical trial of post-operative dietary supplements in surgical patients. Gut 1997;40:393-9.

38 Bastow MD, Rawlings J, Allison SP. Benefits of supplementary tube feeding after fractured neck of femur: a randomised control trial. $B M \mathcal{F}$ 1993;287:1589-92.

39 Delmi M, Rapin CH, Bengoa JM, Delmas PD, Vasey H, Bonjour JP. Dietary supplementation in elderly patients with fractured neck of femur. Lancet 1990;335:1013-6.

40 Von Meyenfeld MF, Meijerink WJHJ, Rouflard MJ, Buil-Maassen NTHJ, Soeters PB. Perioperative nutritional support - a randomised operative nutritional support - a rand
clinical trial. Clin Nutr 1992;11:180-6.

41 Askanazi J, Starker PN, Olsson C, et al. Effect of immediate post-operative nutritional support on the length of hospitalisation. Ann Surg 1986; 203:236-9.

42 Veterans Affairs Total Parenteral Nutrition Cooperative Study Group. Pre-operative total parenteral nutrition in surgical patients. $N$ Engl $\exists$ Med 1991;325:525-32.

43 McWhirter JP, Hill K, Richards J, Pennington $\mathrm{CR}$. The use, efficacy and monitoring of artificial nutritional support in a teaching hospital. Scot Med $\mathcal{f}$ 1995;40:179-83.

44 Jackson AA. Nutrition in the undergraduate curriculum. Proc Nutr Soc 1996;55:775-81.

45 Bower RH, Carra FB, Bershadsky B, et al. Early enteral administration of a formula (Impact[R]) supplemented with arginine, nucleosides, and fish oil in intensive care unit patients:results of a multicentre, prospective randomised clinical multicentre, prospective random 